\title{
SMALL SETS IN CONVEX GEOMETRY AND FORMAL INDEPENDENCE OVER ZFC
}

\author{
MENACHEM KOJMAN
}

Received 28 June 2004

To each closed subset $S$ of a finite-dimensional Euclidean space corresponds a $\sigma$-ideal of sets $\mathscr{F}(S)$ which is $\sigma$-generated over $S$ by the convex subsets of $S$. The set-theoretic properties of this ideal hold geometric information about the set. We discuss the relation of reducibility between convexity ideals and the connections between convexity ideals and other types of ideals, such as the ideals which are generated over squares of Polish space by graphs and inverses of graphs of continuous self-maps, or Ramsey ideals, which are generated over Polish spaces by the homogeneous sets with respect to some continuous pair coloring. We also attempt to present to nonspecialists the set-theoretic methods for dealing with formal independence as a means of geometric investigations.

\section{Introduction}

This paper has two objectives. Its first objective is to present in a unified way a connected group of set-theoretic results about convexity in Euclidean spaces $[4,5,6,10,12]$. These investigations concern a generalization of convexity of closed subsets of Euclidean spaces. We call a set countably convex if it is a countable union of convex sets and uncountably convex otherwise. A convex cover of a set $S$ is a collection of convex subsets of $S$ which covers $S$.

It is fair to say that an uncountably convex set is "less convex" than a countably convex one. But how does one compare generalized convexity between two uncountably convex sets? For every set $S \subseteq \mathbb{R}^{d}$ the collection $\{\{x\}: x \in S\}$ is a convex cover of $S$, so for a subset of $\mathbb{R}^{d}$ it never takes more than continuum many convex subsets to cover it. On the other hand, a closed uncountable subset of $\mathbb{R}^{d}$ is equinumerous with the continuum. So are not all uncountably convex closed subsets of $\mathbb{R}^{d}$ for all $d \geq 1$ "equally nonconvex"?

Most mathematicians would probably think that the answer to this question is positive, but this is not so. For each finite dimension $d \geq 1$ there exists an uncountably convex compact set $S_{d+1} \subseteq \mathbb{R}^{d+1}$ which is "strictly more convex" than every uncountably convex $S \subseteq \mathbb{R}^{d}$, in the following sense:

(i) for every closed uncountably convex $S \subseteq \mathbb{R}^{d}$, there is a uniform way for translating convex covers of $S$ to convex covers of $S_{d+1}$. This makes $S_{d+1}$ "at least as convex" as $S$, 
(ii) there is no proof from the axioms of set theory that for some closed and uncountably convex $S \subseteq \mathbb{R}^{d}$ it holds that $S$ is at least as convex as $S_{d+1}$, because it is possible that $S_{d+1}$ is a union of strictly fewer convex sets than the minimal number of convex subsets of $S$ required to cover $S$ for every closed uncountably convex $S \subseteq \mathbb{R}^{d}[6]$.

The second clause above brings up the second objective of this paper, which is to present the set-theoretic methodology for dealing with possible different uncountable infinities in Euclidean spaces and to demonstrate the applicability of this methodology to geometric investigations. Using the set-theoretic language of formal independence over the axioms of set theory, one can phrase and prove geometric properties of $\mathbb{R}^{d}$ which are neither expressible nor provable otherwise.

In Section 2, the convexity ideal of a closed, uncountably convex subset of $\mathbb{R}^{d}$ is defined and so is the relation of reducibility between $\sigma$-ideals, used to compare different convexity ideals. This relation is a variant of Tukey reducibility (see [2] for a treatment of Tukey reducibility). Then the sets $S_{d} \subseteq \mathbb{R}^{d}$ are constructed for all $d \geq 1$ and are shown to form a descending sequence in the reducibility relation.

The methodology for showing that the reducibility relations which are presented in Section 2 are not reversible is presented in Sections 3 and 4 is devoted to consistency results.

A particularly interesting case is $d=2$. The classification of generalized convexity of closed planar sets leads to some interesting connection to other ideals. Section 5 describes the connection of convexity ideals in the plane to functions ideals, which are generated over squares of Polish spaces by graphs and inverses of graphs of continuous self-maps of the space, to Ramsey ideals, and to the $\sigma$-compact ideal over the Baire space.

\section{Convexity ideals}

\subsection{Ideals and covering}

Definition 2.1. (i) An ideal of sets is a nonempty collection of sets $\mathscr{I}$ which satisfies:

(1) $A, B \in \mathscr{I} \Rightarrow A \cup B \in \mathscr{I}$,

(2) $A \subseteq B \in \mathscr{I} \Rightarrow A \in \mathscr{I}$.

The term "ideal" will always mean "an ideal of sets."

(ii) The domain of an ideal $\mathscr{I}$ is the set $\operatorname{dom} \mathscr{I}:=\bigcup \mathscr{I}$. If $\operatorname{dom} \mathscr{I} \notin \mathscr{I}$, then $\mathscr{I}$ is a proper ideal.

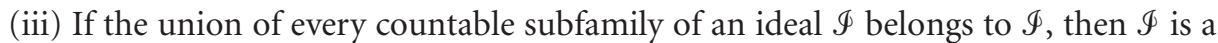
$\sigma$-ideal.

(iv) A subfamily $\mathscr{F} \subseteq \mathscr{I}$ of an ideal $\mathscr{I}$ is a covering family of $\mathscr{I}$ if $\bigcup \mathscr{F}=\operatorname{dom} \mathscr{I}$.

(v) If $\mathscr{F}$ is a family of sets, then the ideal generated by $\mathscr{F}$ is

$$
\left\{X: \exists n \exists\left(A_{1}, \ldots, A_{n}\right)\left[\text { each } A_{i} \in \mathscr{F} \text { and } X \subset \bigcup_{i=1}^{n} A_{i}\right]\right\} .
$$

(vi) The $\sigma$-ideal which is $\sigma$-generated by a family $\mathscr{F}$ of sets is

$$
\left\{X: \exists\left(A_{1}, A_{2}, \ldots\right)\left[\text { each } A_{i} \in \mathscr{F} \text { and } X \subset \bigcup_{i=1}^{\infty} A_{i}\right]\right\} .
$$


In what follows we will be interested in comparing the difficulty of finding covering families of one ideal to that of finding covering families of another. This will be done with the following relation of reducibility.

Definition 2.2. A reduction of an ideal $\mathscr{F}$ to an ideal $\mathscr{I}$ is a function $f: \operatorname{dom} \mathscr{I} \rightarrow \operatorname{dom} \mathscr{F}$ such that $f^{-1}[Y] \in \mathscr{I}$ for all $Y \in \mathscr{F}$ or, equivalently, such that $f[X] \notin \mathscr{\mathscr { S }}$ for all $X \subseteq \operatorname{dom} \mathscr{I}$ such that $X \notin \mathscr{I}$. An ideal $\mathscr{F}$ is reducible to an ideal $\mathscr{I}$ if there exists a reduction $f$ of $\mathscr{F}$ to $\mathscr{I}$. Write $\mathscr{I} \leq \mathscr{F}$ to denote that $\mathscr{F}$ is reducible to $\mathscr{I}$. If both $\mathscr{I} \leq \mathscr{F}$ and $\mathscr{S} \leq \mathscr{I}$ hold, then $\mathscr{I}$ and $\mathscr{S}$ are equivalent.

The relation $\leq$ on ideals is reflexive and transitive, hence equivalence of ideals is an equivalence relation.

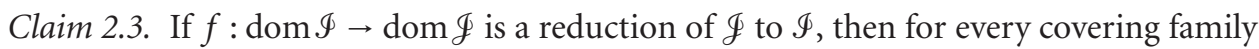
$\mathscr{G} \subseteq \mathscr{F}$ of $\mathscr{F}$, the family $\mathscr{F}:=\left\{f^{-1}[Y]: Y \in \mathscr{G}\right\}$ is a covering family of $\mathscr{I}$.

Proof. Since $f^{-1}[Y] \in \mathscr{I}$ for all $Y \in \mathscr{F}$, indeed $\mathscr{F} \subseteq \mathscr{I}$. To see that $\mathscr{F}$ covers $\operatorname{dom} \mathscr{I}$, let $x \in \operatorname{dom} \mathscr{I}$ be arbitrary. Since $\mathscr{G}$ is covering, there is some $Y \in \mathscr{G}$ so that $f(x) \in Y$; so $x \in f^{-1}[Y]$.

Thus, if $\mathscr{I} \leq \mathscr{S}$, then every reduction $f: \operatorname{dom} \mathscr{I} \rightarrow \operatorname{dom} \mathscr{S}$ gives a uniform way of translating covering families of $\mathscr{I}$ to covering families of $\mathscr{\mathscr { S }}$.

The simplest example of $\mathscr{I} \leq \mathscr{F}$ is when $\mathscr{F} \subseteq \mathscr{I}$ and $\operatorname{dom} \mathscr{\mathscr { F }}=\operatorname{dom} \mathscr{I}$, that is, when $\mathscr{\mathscr { F }}$ is a subideal of $\mathscr{S}$ with the same domain. In this case, the identity function on $\operatorname{dom} \mathscr{I}=\operatorname{dom} \mathscr{F}$ is a reduction of $\mathscr{F}$ to $\mathscr{T}$. Things are more complicated when $\mathscr{\mathscr { S }} \subseteq \mathscr{\mathscr { S }}$ and $\operatorname{dom} \mathscr{F} \neq \operatorname{dom} \mathscr{F}$ : a reduction may or may not exist in either direction.

We will be often considering a special case of this latter situation, when $\mathscr{I}$ is a restriction of $\mathscr{F}$ to a subset $A$ of $\operatorname{dom} \mathscr{F}$.

Definition 2.4. Suppose $\mathscr{F}$ is an ideal and $\varnothing \neq A \subseteq \operatorname{dom} \mathscr{F}$. The ideal $\{X: X \subseteq A, X \in \mathscr{F}\}$ $=\{X \cap A: X \in \mathscr{F}\}$ is denoted by $\mathscr{F} \uparrow A$.

Lemma 2.5. Suppose that $\mathscr{F}$ is an ideal and that $\varnothing \neq A \subseteq \operatorname{dom} \mathscr{F}$. Then $\mathscr{F} \uparrow A \leq \mathscr{J}$. If in addition $\operatorname{dom} \mathscr{F} \backslash A \in \mathscr{F}$ holds, then $\mathscr{I} \leq \mathscr{F} \uparrow A$ holds.

Proof. To see that $\mathscr{F}\lceil A \leq \mathscr{F}$, let $f: A \rightarrow \operatorname{dom} \mathscr{E}$ be the identity map on $A$. For all $Y \in \mathscr{F} \uparrow$ $A$, it holds that $f[Y]=Y$, hence, $Y \notin \mathscr{F} \uparrow A \Rightarrow f[Y] \notin \mathscr{J}$. This shows that $f$ is a reduction of $\mathscr{F}$ to $\mathscr{F} \uparrow A$.

Assume now that $\operatorname{dom} \mathscr{F} \backslash A \in \mathscr{F}$. Let $f: \operatorname{dom} \mathscr{F} \rightarrow A$ be any projection, that is, an onto function which satisfies $f^{2}=f$. Suppose $Y \subseteq \operatorname{dom} \mathscr{E}$ and $Y \notin \mathscr{F}$. It holds that $Y=$ $(Y \cap A) \cup(Y \backslash A)$ and, since $(Y \backslash A) \subseteq(\operatorname{dom} \mathscr{F} \backslash A) \in \mathscr{E}$, we have $(Y \backslash A) \in \mathscr{F}$. Therefore, since $Y \notin \mathscr{F}$, necessarily $(Y \cap A) \notin \mathscr{F}$. But $(Y \cap A) \subseteq f[Y]$, hence $f[Y] \notin \mathscr{F}\lceil A$, so $f$ is a reduction of $\mathscr{E} \uparrow A$ to $\mathscr{E}$.

Definition 2.6. A topological space $X$ is Polish if it is homeomorphic to a complete metric space. A topological space $X$ is perfect if it has no isolated points. For a perfect Polish space $X$ let $M(X)$ denote the meager, or first category, $\sigma$-ideal over $X$, namely the $\sigma$-ideal which is $\sigma$-generated by all (closed) nowhere dense subsets of $X$. 
If $X$ is a perfect Polish space, then, since $X$ has no isolated points, each singleton $\{x\}$ for $x \in X$ belongs to $\mathcal{M}(X)$, hence $\operatorname{dom} M(X)=X$. By the previous lemma, whenever $A \subseteq X$ is a dense $G_{\delta}$ subset of a perfect Polish space $X$, it holds that $\mathcal{M}(X)$ and $\mathcal{M}(X)\lceil A$ are equivalent.

Claim 2.7. All meager ideals over all perfect Polish spaces are equivalent to each other.

Proof. Suppose $X$ and $Y$ are perfect Polish spaces and fix countable dense sets $A \subseteq X$ and $B \subseteq Y$. Since $X$ and $Y$ are perfect, both $A$ and $B$ are dense in themselves and thus homeomorphic to each other. Fix a homeomorphism $f^{\prime}: A \rightarrow B$. By Lavrentiev's theorem (see [9, Theorem 3.9]), there are $G_{\delta}$ sets $A^{\prime} \subseteq X, B^{\prime} \subseteq Y$ so that $A \subseteq A^{\prime}, B \subseteq B^{\prime}$ and a homeomorphism $f: A^{\prime} \rightarrow B^{\prime}$ (which extends $f^{\prime}$, but this is not needed here). The homeomorphism $f$ and its inverse $f^{-1}$ demonstrate the equivalence of $M(Y) \uparrow B$ with $\mathcal{M}(X)\lceil A$. Since $\mathcal{M}(X) \uparrow A$ is equivalent to $\mathcal{M}(X)$ and $\mathcal{M}(Y) \uparrow B$ is equivalent to $\mathcal{M}(Y)$, the equivalence of $\mathcal{M}(Y)$ with $\mathcal{M}(X)$ follows.

Since up to equivalence there is just one meager ideal over a perfect Polish space, we use the symbol $\mathcal{M}$ alone to denote the meager ideal.

2.2. Convexity ideals. A subset of a real vector space is countably convex if it is a union of countably many convex sets and is uncountably convex otherwise. Let $d \geq 1$ be a natural number and let $\mathbb{R}^{d}$ denote the $d$-dimensional Euclidean space with the usual Euclidean norm.

Definition 2.8. Suppose $S \subseteq \mathbb{R}^{d}$. Let $\mathscr{F}(S)$ be the $\sigma$-ideal which is $\sigma$-generated over $S$ by all convex subsets of $S$. Explicitly,

$$
X \in \mathscr{F}(S) \Longleftrightarrow \exists\left(c_{0}, c_{1}, \ldots\right)\left[\text { each } c_{i} \subseteq S \text { is convex and } X \subseteq \bigcup_{i} c_{i}\right] .
$$

The $\sigma$-ideal $\mathscr{F}(S)$ is proper if and only if $S$ is uncountably convex. In the case that $S$ is closed, the closure in $\mathbb{R}^{d}$ of a convex subset of $S$ is contained in $S$, and is convex. Thus, when $S$ is closed, $\mathscr{S}(S)$ is $\sigma$-generated by the closed convex subsets of $S$.

Claim 2.9. Suppose $S \subseteq \mathbb{R}^{d}$. Then the union of all relatively open subsets of $S$ which belong to $\mathscr{F}(S)$ belongs to $\mathscr{F}(S)$.

Proof. Let $A:=\bigcup\left\{u \cap S: u \subseteq \mathbb{R}^{d}\right.$ is open and $\left.u \cap S \in \mathscr{J}(S)\right\}$. $A$ is an open subset of $S$. We need to show that $A \in \mathscr{L}(S)$.

For every $x \in A$ there is some open set $u \subseteq \mathbb{R}^{d}$ so that $x \in u$ and $u \cap S \in \mathscr{F}(S)$. Since $u$ is open and $x \in u$, there is some rational ball $B$, that is a ball of rational radius and rational coordinates of its center, so that $x \in B \subseteq u$, and hence $B \cap S \in \mathscr{F}(S)$.

We have shown that $A=\bigcup\{B \cap S: B$ is a rational ball and $B \cap S \in \mathscr{F}(S)\}$. For each rational ball $B$ which satisfies $B \cap S \in \mathscr{F}(S)$ fix convex subsets of $S, c_{0}^{B}, c_{1}^{B}, \ldots$, so that $B \cap S \subseteq$ $\bigcup_{i} c_{i}^{B}$.

Now

$$
\begin{aligned}
A & =\bigcup\{B \cap S: B \text { is a rational ball and } B \cap S \in \mathscr{F}(S)\} \\
& \subseteq \bigcup\left\{c_{i}^{B}: B \text { is a rational ball and } B \cap S \in \mathscr{F}(S)\right\} .
\end{aligned}
$$


Since there are only countably many rational balls in $\mathbb{R}^{d}$, the collection $\left\{c_{i}^{B}: B\right.$ is a rational ball and $B \cap S \in \mathscr{F}(S)\}$ is countable, and so $A$ is contained in a countable union of convex subsets of $S$. Thus $A \in \mathscr{J}(S)$.

Definition 2.10. For $S \subseteq \mathbb{R}^{d}$ let $A(S)$ be the union of all relatively open sets in $\mathscr{I}(S)$ and let $K(S):=S \backslash A(S)$.

Claim 2.11. Suppose $S \subseteq \mathbb{R}^{d}$ is closed and uncountably convex. Then $K(S)$ is nonempty and perfect.

Proof. Since $A(S) \in \mathscr{L}(S)$ and $\mathscr{L}(S)$ is proper, $A(S) \neq S$ hence $K(S) \neq \varnothing$. Since $A(S)$ is open in $S, K(S)$ is closed in $S$, and, since $S$ is closed, $K(S)$ is closed in $\mathbb{R}^{d}$. We argue that no point $x \in K(S)$ is isolated in $K(S)$. Suppose to the contrary that $s \in K(S)$ and an open $u \ni x$ are given so that $K(S) \cap u=\{x\}$. Then $(u \cap S) \backslash\{x\} \subseteq A(S)$ and hence it belongs to $\mathscr{E}(S)$. Since $\{x\}$ is convex, also $(u \cap S) \backslash\{x\} \cup\{x\}=u \cap S$ belongs to $\mathscr{E}(S)$. Thus, $u \cap S \subseteq A(S)$. But since $x \in S \backslash A(S)$, this is a contradiction.

Definition 2.12 (the convexity ideal of a closed uncountably convex set). Suppose that $S \subseteq \mathbb{R}^{d}$ is closed and uncountably convex. Let $\mathscr{I}(S)$ be the ideal $\mathscr{\mathscr { S }}(S) \uparrow K(S)=\{X \cap K(S)$ : $X \in \mathscr{J}(S)\}$. Equivalently, $\mathscr{I}(S)$ is the $\sigma$-ideal $\sigma$-generated over $K(S)$ by all intersections $C \cap K(S)$ of all closed and convex $C \subseteq S$ with $K(S)$. The ideal $\mathscr{S}(S)$ is called the convexity ideal of $S$.

Claim 2.13. For a closed uncountably convex $S \subseteq \mathbb{R}^{d}$, the ideals $\mathscr{\mathscr { S }}(S)$ and $\mathscr{\mathscr { S }}(S)$ are equivalent.

Proof. By Lemma 2.5, since $S \backslash K(S)=A(S) \in \mathscr{L}(S)$.

Claim 2.14. Suppose $S \subseteq \mathbb{R}^{d}$ is closed and uncountably convex. Then for every convex $C \subseteq S$, the intersection $C \cap K(S)$ is nowhere dense in $K(S)$.

Proof. Suppose to the contrary that $C \subseteq S$ is convex and that $C \cap K(S)$ is dense in $K(S) \cap$ $u$ for some open $u$ with $u \cap K(S) \neq \varnothing$. Since the closure in $\mathbb{R}^{d}$ of $C$ is convex and is contained in $S, K(S) \cap u \in \mathscr{F}(S)$ and hence $S \cap u \in \mathscr{F}(S)$. This contradicts $A(S) \cap K(S)=$ $\varnothing$.

Corollary 2.15. For a closed, uncountably convex $S \subseteq \mathbb{R}^{d}$, it holds that $\Phi(S) \subseteq \mathcal{M}(K(S))$ and that $\operatorname{dom} \Phi(S)=\operatorname{dom} M(K(S))=K(S)$. Thus, $M \leq \mathscr{I}(S)$ for every closed, uncountably convex $S \subseteq \mathbb{R}^{d}$ for all $d \geq 1$.

Proof. If $X \subseteq S$ is countably convex and $X \subseteq \bigcup_{n} c_{n}$, where each $c_{n} \subseteq S$ is convex, then $X \cap$ $K(S) \subseteq \bigcup_{n} c_{n} \cap K(S)$. By the previous claim, $c_{n} \cap K(S)$ is nowhere dense in $K(S)$ for each $n$, hence $X \cap K(S) \in \mathcal{M}(K(S))$. This shows the $\mathscr{I}(S) \subseteq \mathcal{M}(K(S))$. Also, every singleton in $K(S)$ is a convex set, hence $\operatorname{dom} \mathscr{I}(S)=K(S)$. Thus $\mathscr{I}(S)$ is a subideal of $\mathcal{M}(K(S))$ with the same domain, and is reducible to it.

A covering family of $\mathscr{I}(S)$, for a closed and uncountably convex $S \subseteq \mathbb{R}^{d}$, corresponds naturally to a covering of $S$ by convex subsets. The reducibility $M \leq \Phi(S)$ which was just established shows that to cover a closed, uncountably convex set by convex subsets is at least as hard as covering a perfect Polish space by nowhere dense sets. 
2.3. The structure of convexity ideals under reducibility. In this section, we will examine the relation of reducibility on convexity ideals. We will construct, for each $d \geq 1$, a closed and uncountably convex set $S_{d} \subseteq \mathbb{R}^{d}$ whose convexity ideal $\mathscr{S}\left(S_{d}\right)$ is identical with a combinatorially described ideal $\mathscr{I}_{d}$. We will see that for every closed, uncountably convex $S \subseteq \mathbb{R}^{d}$, it holds that

$$
\mathscr{I}_{d+1}=\mathscr{I}\left(S_{d+1}\right) \leq \mathscr{I}(S) .
$$

We first describe the ideals $\mathscr{I}_{d}$ combinatorially, using the standard metric on spaces of sequences, and prove that $\mathscr{I}_{d+1} \leq \mathscr{I}_{d}$ for all $d \geq 1$. Then we show how to realize each ideal $\Phi_{d}$ as a convexity ideal of a compact subset of $\mathbb{R}^{d}$. The concatenation of a sequence $v$ to a sequence $\eta$ is denoted by $\eta \hat{\nu}$.

For every $d \geq 2$, let $d^{\mathbb{N}}$ denote the space of all sequences over the $d$ distinct symbols $0,1, \ldots, d-1$. Let $\langle\cdot\rangle$ denote the empty sequence, let $\left\langle x_{0}, \ldots, x_{n-1}\right\rangle$ denote a sequence of length $n$, and let $\left\langle x_{m}: m \in \mathbb{N}\right\rangle$ denote an infinite sequence.

For distinct $\eta, v \in d^{\mathbb{N}}$ let $\Delta(\eta, v):=\min \{n: \eta(n) \neq \nu(n)\}$ and let $\rho(\eta, v):=1 / 2^{\Delta(\eta, v)}$ (and let $\rho(\eta, \eta)=0$ for all $\eta$ ). The function $\rho$ is a metric with which $d^{\mathbb{N}}$ is a compact metric space. There are equidistant sets of size $d$ in $d^{\mathbb{N}}$ with respect to $\rho$ but no equidistant sets of size $d+1$. An open ball with center $\eta$ is the set of all $v$ so that $\Delta(\eta, v) \geq k$ for some constant $k$. If a set $X \subseteq d^{\mathbb{N}}$ is somewhere dense in $d^{\mathbb{N}}$, it must contain an equidistant subset of size $d$.

Definition 2.16. For every $d \geq 3$, let $\Phi_{d}$ be the $\sigma$-ideal over $d^{\mathbb{N}}$ which is $\sigma$-generated by all subsets which do not contain a set of $d$ equidistant points.

The ideal $\Phi_{d}$ is contained in $\mathcal{M}\left(d^{\mathbb{N}}\right)$ for all $d \geq 3$ by what we observed.

We remark that it makes sense to apply the definition of $\Phi_{d}$ also to $d=2$-one gets in this case the $\sigma$-ideal of countable subsets of $2^{\mathbb{N}}$. However, we denote the $\sigma$-ideal of countable subsets of $2^{\mathbb{N}}$ by $\Phi_{1}$ and reserve the notation $\Phi_{2}$ for the following ideal.

Definition 2.17. Let $\Phi_{2}$ be the $\sigma$-ideal which is $\sigma$-generated over $2^{\mathbb{N}}$ by all subsets $X \subseteq 2^{\mathbb{N}}$ which satisfy the following: $\Delta(\eta, \nu)$ is even for all distinct $\eta, \nu \in X$, or $\Delta(\eta, \nu)$ is odd for all distinct $\eta, v$ in $X$.

The following claim follows from Theorem 2.20 and Claim 2.19 below; but it also has a direct, short proof.

Claim 2.18. $\mathscr{I}_{d+1} \leq \mathscr{I}_{d}$ for all $d \geq 1$.

Proof. The identity map on $2^{\mathbb{N}}$ is a reduction of $\mathscr{I}_{1}$ to $\mathscr{I}_{2}$, since every countable subset of $2^{\mathbb{N}}$ belongs to $\Phi_{2}$.

A reduction of $\mathscr{I}_{2}$ to $\mathscr{I}_{3}$ is achieved as follows: let $g(0)=\langle 0,0\rangle, g(1)=\langle 1,0\rangle$, and $g(2)=\langle 1,1\rangle$. Now define $f(\eta)=\langle g(\eta(m)): m \in \mathbb{N}\rangle$, that is, the successive concatenation of $g(\eta(i))$. Suppose that $\left\{\eta_{0}, \eta_{1}, \eta_{2}\right\}$ is an equidistant triple in $3^{\mathbb{N}}$ with $\Delta\left(\eta_{i}, \eta_{j}\right)=m$ for all $i<j<3$ and suppose that $\eta_{i}(m)=i$. Then $\Delta\left(f\left(\eta_{0}, f \eta_{1}\right)\right)=2 m$ and $\Delta\left(f\left(\eta_{1}\right), f\left(\eta_{2}\right)\right)=$ $2 m+1$. Therefore, whenever in $Y \subseteq 2^{\mathbb{N}}$ all $\Delta$ 's are odd or all $\Delta$ 's are even, $f^{-1}[Y]$ does not contain an equidistant triple. Since $f^{-1}$ (on sets) commutes with taking unions, for every $Y \in \mathscr{I}_{2}$, it holds that $f^{-1}[Y] \in \mathscr{I}_{3}$. 
For the case $d \geq 3$, let $g(i)=i$ if $i<d$ and let $g(d)=d-1$. Define $f(\eta)=\langle g(\eta(m))$ : $m \in \mathbb{N}\rangle$. Every equidistant $(d+1)$-tuple in $(d+1)^{\mathbb{N}}$ is mapped via $f$ to an equidistant $d$-tuple. Thus, $f$ is a reduction of $\mathscr{I}_{d}$ to $\Phi_{d+1}$.

Claim 2.19. For every closed and uncountably convex $S \subseteq \mathbb{R}^{d}$, it holds that $\mathscr{I}_{d+1} \leq \mathscr{I}(S)$.

Proof. A uniformly continuous 1-1 function $f:(d+1)^{\mathbb{N}} \rightarrow K(S)$ is defined as follows. For every finite sequence $\eta \in(d+1)^{m}$, define $F(\eta) \subseteq K(S)$ to be a closed ball of positive radius less than or equal to $1 /(m+1)$ and so that $F\left(\eta^{\wedge} i\right) \subseteq F(\eta)$ for all $i \leq d$. Then $f(\eta)$ for an infinite $\eta \in(d+1)^{\mathbb{N}}$ is defined as the unique point which satisfies $\{f(\eta)\}=\bigcap_{m} F(\eta\lceil m)$.

Let $F(\langle\cdot\rangle)$ be any ball of radius 1 in $K(S)$.

Suppose $F(\eta)$ is defined for all $\eta \in(d+1)^{m}$ and let $\eta \in(d+1)^{m}$ be given. Since $\operatorname{conv}(K(S) \cap F(\eta) \nsubseteq S$, it follows, by Caratheodory's theorem (Caratheodory's theorem states that a point in the convex hull of $S \subseteq \mathbb{R}^{d}$ belongs to the convex hull of $d+1$ points from $S)$, that there are $y_{0}, \ldots, y_{d} \in K(S) \cap F(\eta)$ so that $\operatorname{conv}\left(y_{0}, \ldots, y_{d}\right) \nsubseteq S$. Since $S$ is closed, there is a sufficiently small $r>0$ so that for every choice of $x_{i} \in B\left(y_{i}, r\right)$ for $i \leq d$, it holds that $\operatorname{conv}\left(x_{0}, \ldots, x_{d}\right) \nsubseteq S$. Let $f\left(\eta^{\wedge} i\right)=B\left(y_{i}, r\right)$.

Every equidistant $(d+1)$-tuple in $(d+1)^{\mathbb{N}}$ is mapped via $f$ to a $(d+1)$-tuple in $K(S)$ whose convex hull is not contained in $S$. Thus, $f$ is a reduction.

Finally, we show that $\Phi_{d}$ is realized as a convexity ideal in $\mathbb{R}^{d}$.

Theorem 2.20. For every $d \geq 1$, there exists a compact set $S_{d} \subseteq \mathbb{R}^{d}$ so that $\Phi(S)=\Phi_{d}$. For $d \geq 3, S_{d}$ is star-like with respect to a point in its interior, namely there is some $x_{0} \in \operatorname{int} S_{d}$ so that $\left[x_{0}, y\right] \subseteq S_{d}$ for all $y \in S_{d}$, and therefore $S_{d}$ is contractible for all $d \geq 3$.

Proof. We start with the case $d=3$. Take a spherical soup bowl and place three closed spherical caps and an open pyramid on its bottom so that

(1) any point in one of the spherical caps can see any point in any other spherical cap;

(2) any choice of one point from each cap spans a triangle that meets the pyramid. (See Figure 2.1.)

Repeat this construction ad infinitum inside each of the three caps. When finished, fill the bowl with soup and freeze it. The frozen soup is a compact subset of $\mathbb{R}^{3}$ which we denote by $S_{3}$. Clearly, $S_{3}$ is star-like with respect to the center of the sphere, which belongs to the interior of $S_{3}$.

The set $K\left(S_{3}\right)$ consists of all points in $S_{3}$ which belong to infinitely many caps. The set $K\left(S_{3}\right)$ is thus naturally homeomorphic to $3^{\mathbb{N}}$. Whenever $X \subseteq 3^{\mathbb{N}}$ does not contain an equidistant triple, it corresponds via the natural homeomorphism to a subset of $K(S)$ whose convex hull is contained in $S$. If $X$ does contain an equidistant triple, then their corresponding points in $K(S)$ are in three caps that separated from each other simultaneously at some stage of the construction, so their convex hull meets one of the pyramids, and is thus not contained in $S_{3}$. We have shown that $\Phi\left(S_{3}\right)=\Phi_{3}$.

A straightforward generalization of this construction gives a contractible, compact $S_{d} \subseteq \mathbb{R}^{d}$ so that $\Phi\left(S_{d}\right)=\Phi_{d}$ for all $d \geq 3$.

In $\mathbb{R}^{2}$ the construction is different. Let $C$ denote the standard middle-third Cantor set, namely, the set of all points in $[0,1]$ whose base- 3 expansion omits the digit 1 . Fix 


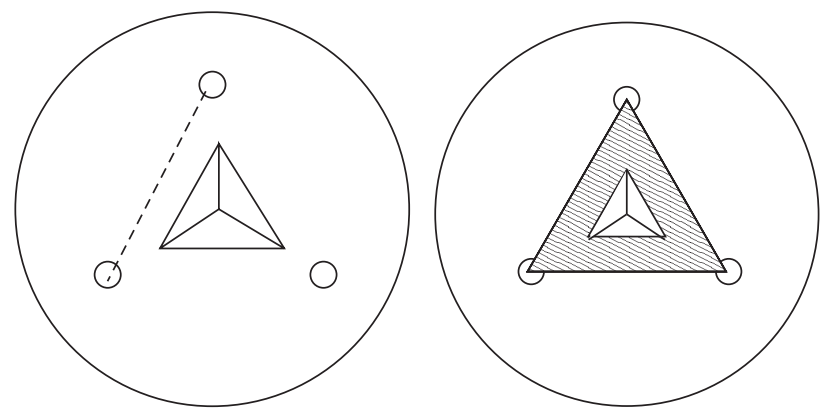

Figure 2.1

a continuously differentiable function $f: C \rightarrow \mathbb{R}$ with the following property: for all $x_{1}<$ $x_{2}$ in $C$,

(1) $f^{\prime}\left(x_{1}\right)<\left(f\left(x_{2}\right)-f\left(x_{1}\right)\right) /\left(x_{2}-x_{1}\right)<f^{\prime}\left(x_{2}\right)$ if and only if the first position in which $x_{1}$ and $x_{2}$ have different digits in their base- 3 expansions is even,

(2) $f^{\prime}\left(x_{1}\right)>\left(f\left(x_{2}\right)-f\left(x_{1}\right)\right) /\left(x_{2}-x_{1}\right)>f^{\prime}\left(x_{2}\right)$ if and only if the first position in which $x_{1}$ and $x_{2}$ have different digits in their base- 3 expansions is odd.

Such a function can be defined as a uniformly continuous limit of piece-wise linear functions.

Let $S_{2}$ be the union of all convex hulls of triangles $\left\{\left(x_{1}, f\left(x_{1}\right)\right),\left(x_{2}, f\left(x_{2}\right)\right),\left(x_{3}, f\left(x_{3}\right)\right)\right\}$ for $x_{1}<x_{2}<x_{3}$ in $C$ for which either all three pairs $\left(x_{i}, x_{j}\right)$ for $0<i<j<3$ satisfy condition (1) or all three pairs satisfy condition (2) above. Call such a triangle homogeneous.

It is not hard to verify that $S_{2}$ is closed, and uncountably convex and that $K(S)$ is exactly the graph of $f$, and is thus naturally homeomorphic to $2^{\mathbb{N}}$. Furthermore, the convex hull of a subset of $K(S)$ is contained in $S$ if and only if all triangles from the subset are homogeneous, which means that the set itself is homogeneous. This establishes that $\mathscr{I}\left(S_{2}\right)=\mathscr{I}_{2}$.

Finally, $\Phi_{1}$, the ideal of countable subsets of $2^{\mathbb{N}}$, is the convexity ideal of the standard Cantor set.

We point out the following.

Fact 2.21. If $S \subseteq \mathbb{R}^{1}$ is closed and uncountably convex, then $\Phi_{1} \leq \mathscr{I}(S)$.

Thus, up to equivalence, there is exactly one convexity ideal of a closed subset of $\mathbb{R}$.

The following pattern of reducibility among convexity ideals is seen now in Figure 2.2: there is a single ideal in $\mathbb{R}^{1}$. In each dimension $d+1$ there is single set $S_{d+1}$ so that all convexity ideals in $\mathbb{R}^{d}$ are reducible to its convexity ideal, $\Phi_{d+1}$. All convexity ideals are reducible to the meager ideal.

\section{Expanding the methodology: proofs, cardinals, models, and consistency}

If one wishes to know the structure of reducibility among convexity ideals up to equivalence, then one must answer the following. 


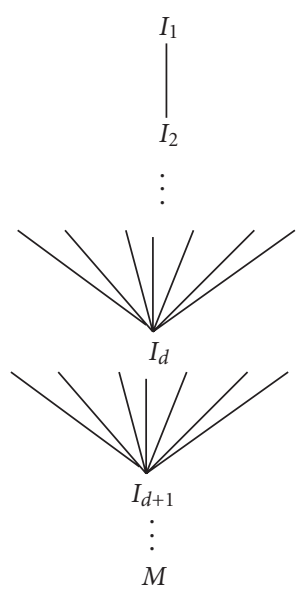

Figure 2.2

Question 3.1. Which of the reducibility relations in Figure 2.2 are reversible?

This seemingly innocent question involves formal independence over the axioms of set theory. The usual axioms of mathematics do not decide the reversibility or irreversibility of any of the reducibilities in this figure. The next section will present the relevant facts about formal independence.

Meanwhile we begin by showing that if one assumes the continuum hypothesis as an additional axiom, then all convexity ideals (and many other ideals) are equivalent to each other, and thus, with the $\mathrm{CH}$, the whole figure collapses to a single point modulo equivalence. Since the $\mathrm{CH}$ is a consistent axiom [6], this shows the inability to prove without additional assumptions that there are two inequivalent convexity ideals.

Theorem 3.2. If the continuum hypothesis holds, then every proper $\sigma$-ideal on the continuum which is $\sigma$-generated by continuum many generators is reducible to $\mathscr{I}_{1}$, the ideal of countable subsets of $2^{\mathbb{N}}$.

Proof. Assuming $\mathrm{CH}$, we identify the continuum with the ordinal $\omega_{1}=\left\{\alpha: \alpha<\omega_{1}\right\}$. Given a proper $\sigma$-ideal $\mathscr{F}$ with $\operatorname{dom} \mathscr{F}=\omega_{1}$ which is $\sigma$-generated by $\omega_{1}$ generators, fix an enumeration $\left\langle X_{\alpha}: \alpha<\omega_{1}\right\rangle$ of a set of generators of $X$.

Now for each $\alpha<\omega_{1}$, let $f(\alpha):=\min \left(\omega_{1} \backslash \bigcup_{\gamma \leq \alpha} X_{\gamma}\right)$. Since $\mathscr{F}$ is a proper $\sigma$-ideal, $\omega_{1} \backslash$ $\bigcup_{\gamma \leq \alpha} X_{\gamma}$ is nonempty, and the definition makes sense.

Suppose that $Y \in \mathscr{L}$. Since $\left\{X_{\alpha}: \alpha<\omega_{1}\right\} \sigma$-generates $\mathscr{F}$, there is a countable set $\left\{\alpha_{n}\right.$ : $n \in \mathbb{N}\} \subseteq \omega_{1}$ so that $A \subseteq \bigcup_{n} X_{\alpha_{n}}$. Every countable subset of $\omega_{1}$ is bounded in $\omega_{1}$, hence $\alpha=\sup \left\{\alpha_{n}: n \in \mathbb{N}\right\}<\omega_{1}$. Thus, for all $\alpha<\beta<\omega_{1}$ it holds that $f(\beta) \notin Y$. In other words, $f^{-1}[Y] \subseteq \alpha$, and is therefore countable. Thus $f$ is a reduction of $\mathscr{E}$ to the ideal of countable subsets of $\omega_{1}$.

Corollary 3.3. The CH implies that all convexity ideals, the meager ideal, and the Lebesgue null ideal are equivalent to the ideal of countable sets, and therefore to each other. 
Proof. The ideal of countable sets is trivially reducible to each convexity ideal, to the meager ideal, and to the Lebesgue null ideal. Since each convexity ideal in question is $\sigma$-generated by closed sets, and there are exactly continuum many closed subsets of any perfect Polish space, each of these ideals is $\sigma$-generated by continuum many generators, and by the previous theorem is reducible to the ideal of countable sets under $\mathrm{CH}$.

We will argue soon that it is impossible to prove that $\mathscr{I}_{d} \leq \mathscr{I}_{d+1}$ for any $d \geq 1$. This will necessitate a broadening of the methodology. We will need to make precise what a proof is and how one shows that a certain statement has no proof. We will also introduce the notion of a cardinal invariant.

3.1. What is a proof? We adopt the notion of a formal proof from the Zermelo-Fraenkel axioms of set-theory with the axiom of choice (ZFC). A formal proof from a set of axioms in some formal language $\mathscr{L}$ is a sequence of formulas, each of which is either an axiom in the set or is derived from earlier formulas in the sequence by some logical inference rule. A formula with no free variables is a sentence. A proof of a sentence $\phi$ is a proof whose last formula is $\phi$. This (Hilbertian) notion of formal proof encompasses all proof methods which are accepted by mathematicians. A formal proof itself is a finite string of symbols. Now that a formal proof is a finite mathematical object, the existence or nonexistence of a certain formal proof can be discussed mathematically.

The ZFC set of axioms are sentences in the language of set theory, which contains, apart from the logical symbols $\forall, \exists, \wedge, \vee, \rightarrow, \neg$, the relation symbols $=$ and $\in$. ZFC is the standard axiomatization of set theory. Within ZFC all of mathematics can be formalized. By this we mean the following: every mathematical object and relation can be represented as a set and every proof can be represented as a formal proof from the ZFC axioms.

We need now to clarify how one proves that a proof of a certain sentence from ZFC does not exist. This can be true only if a contradiction cannot be proved from ZFC, since the inference rules easily allow one to produce a proof of any sentence from a proof of a contradiction. A set of axioms from which no contradiction is provable is called consistent.

However, by Gödel's second incompleteness theorem, from ZFC itself there is no proof that ZFC is consistent-unless ZFC is inconsistent, in which case everything is provable from ZFC (including the statement that ZFC is consistent).

We assume from now on that ZFC is consistent. This assumption itself states that some sentences do not have a proof from ZFC; now it remains to find out which ones.

3.2. What is a model? Although a proof is a syntactic object, the usual method for showing that a certain proof does not exist is semantic. A model of ZFC is a set over which the relations $=$ and $\in$ are interpreted, and which satisfies all the axioms of ZFC.

Kurt Gödel proved his completeness theorem in his PhD thesis under Hahn in Vienna in 1930 .

Theorem 3.4. Gödel [4] A sentence $\phi$ is formally provable from a set of axioms $\Gamma$ if and only if $\phi$ holds true in every model of $\Gamma$.

From Gödel's completeness theorem, a necessary and sufficient condition for the nonexistence of a proof of a sentence $\varphi$ from ZFC is the existence of a model of ZFC 
in which $\varphi$ does not hold. In Section 3.5 the method of forcing for constructing models of ZFC is briefly described.

3.3. Infinite cardinals. Recall the notion of an infinite cardinal. An infinite cardinal is an ordinal which does not have a bijection with a smaller ordinal. The cardinality of a set $A$, denoted by $|A|$ is the unique cardinal number with which $A$ has a bijection. For two sets $A, B$, it holds that $|A| \leq|B|$ if and only if there is an injection from $A$ to $B$ if and only if there is a surjection from $B$ onto $A$. Any two infinite cardinals are comparable; equivalently, for any two sets $A, B$, either there is a bijection from $A$ to $B$ or there is a bijection from $B$ to $A$. The infinite cardinals are well ordered, so every nonempty set of infinite cardinals has a smallest member. All these facts about cardinals are ZFC theorems.

The cardinality of $\mathbb{N}$ is the smallest infinite cardinal, named $\Sigma_{0}$. Every infinite cardinal has an immediate successor, and the immediate successor of $\aleph_{0}$ is $\aleph_{1}$, its immediate successor is $\aleph_{2}$ and so forth. Cantor proved that $|\mathbb{R}|>\aleph_{0}$ and conjectured that $|\mathbb{R}|=\aleph_{1}$. The statement $|\mathbb{R}|=\aleph_{1}$ is known as the continuum hypothesis and was presented as the first problem in Hilbert's list of problems in 1900. An equivalent formulation of the continuum hypothesis is that "every set $A \subseteq \mathbb{R}$ is either countable or equinumerous with $\mathbb{R}$."

In 1936, Gödel proved that the continuum hypothesis could not be formally refuted from ZFC (see the 1940 monograph [6]), by providing a model of ZFC in which the statement $|\mathbb{R}|=\aleph_{1}$ holds true.

In 1963, Paul Cohen invented the method of forcing for constructing models of ZFC, and using forcing he constructed a model in which $|\mathbb{R}|=\aleph_{2}$, namely in which the $\mathrm{CH}$ is false. Thus, Cohen showed that $\mathrm{CH}$ could not be proved from ZFC.

3.4. Cardinal invariants. A cardinal invariant is a definition of a cardinal number associated with a structure. The simplest example of a cardinal invariant is $|\mathbb{R}|$ — the cardinality of the real line. Gödel's and Cohen's results showed that it is impossible to determine $|\mathbb{R}|$ from the ZFC axioms.

There are many cardinal invariants associated with $\mathbb{R}$ (a good survey of such invariants is [2]). We will concentrate on the following.

Definition 3.5. Suppose $\mathscr{I}$ is a $\sigma$-ideal over a perfect Polish space. Let $\operatorname{cov}(\mathscr{I})$ be the least cardinality of a covering family $\mathscr{F} \subseteq \mathscr{I}$.

Claim 3.6. Suppose that $\mathscr{F}$ is reducible to $\mathscr{I}$. Then $\operatorname{cov}(\mathscr{I}) \leq \operatorname{cov}(\mathscr{F})$.

Proof. Suppose that $f: \operatorname{dom} \mathscr{I} \rightarrow \operatorname{dom} \mathscr{S}$ is a reduction. By Claim 2.3, for every covering family $\mathscr{G} \subseteq \mathscr{F}$ it holds that $\mathscr{F}:=\left\{f^{-1}[Y]: Y \in \mathscr{G}\right\}$ is a covering family of $\mathscr{\mathscr { S }}$, and clearly $|\mathscr{F}| \leq|\varphi|$.

3.5. The method of forcing and $\sigma$-ideals. Cohen's forcing is a method for extending a given model of ZFC to a larger model of ZFC which contains a new object. A good analogy for forcing is extending a field to a larger field in which a given polynomial gains a new root. The polynomial is a finite description of the new root with coefficients in the old field. After adding a root of a polynomial to the field, one must add all its sums and products with old members of the field to obtain a field. 
In forcing, it is a model of all ZFC which is extended. The description of the new object is not finite, but is a directed set of partial descriptions, called forcing conditions, which belong to the old model.

In Cohen's original forcing (which was designed to produce a model with the negation of $\mathrm{CH}$ ) as well as in many other forcing notions, the forcing conditions are elements of a $\sigma$-ideal over $\mathbb{R}$. The new object which the forcing adds is a real number. The partial description which is provided about the new real by a member of the ideal is that the real avoids it. A Cohen real is added by the Cohen forcing, whose conditions are all closed and nowhere dense subsets of $\mathbb{R}$ which belong to the ground mode, and thus a Cohen real is a new real which avoids all ground model nowhere dense sets.

A later forcing, due to Solovay, adds a new real which avoids all ground model null sets. Such a real is called a random real.

Recall that the real line is a union of two disjoint sets, $\mathbb{R}=A \cup B$, where $A$ is meager and $B$ is null. A Cohen real falls always into $B$ and into all translates of $B$, and thus makes, in the extension, the set of old reals contained in a translate of a null set, and it is therefore null. A random real does the opposite: it turns the set of old reals into a meager set.

Thus, starting from a model of $\mathrm{CH}$ and adding $\mathrm{N}_{2}$ Cohen reals iteratively, one obtains a model in which $\mathbb{R}$ is coverable by $\kappa_{1}$ Lebesgue null sets, but is not coverable by fewer than $\aleph_{2}$ meager sets. Conversely, the iterative addition of $\aleph_{2}$ random reals to a model of $\mathrm{CH}$ produces a model in which $\mathbb{R}$ is coverable by $\mathrm{N}_{1}$ meager sets but not by fewer than $\aleph_{2}$ Lebesgue null sets.

Let $\mathcal{M}$ denote the $\sigma$-ideal of meager subsets of $\mathbb{R}$ and let $\mathcal{N}$ denote the ideal of Lebesgue null subsets of $\mathbb{R}$. The facts in the previous paragraph tell us the following.

THeOREM 3.7. If ZFC is consistent, there is no proof from ZFC that $\mathcal{N}$ is reducible to $M$ and there is no proof from $Z F C$ that $M$ is reducible to $\mathcal{N}$.

Proof. Suppose there was a proof that $\mathcal{N} \leq \mathcal{M}$. Then by Claim 3.6 we have a proof that $\operatorname{cov}(\mathcal{N}) \leq \operatorname{cov}(\mathcal{M})$. But then this inequality has to be true in all models of ZFC—contrary to the existence of a model in which $\operatorname{cov}(\mathcal{N})=\aleph_{2}>\aleph_{1}=\operatorname{cov}(\mathcal{M})$.

Similarly, the other direction is proved.

3.6. Equality, incomparability, inequality, and irreversible inequality. Now comes the main methodological point regarding the comparison of cardinal invariants.

Definition 3.8. Suppose that $\mathscr{I}$ and $\mathscr{\Phi}$ are $\sigma$-ideals which are $\sigma$-generated by some definable collection of closed subsets of a Polish space. Write

$$
\operatorname{cov}(\mathscr{I}) \prec \operatorname{cov}(\mathscr{F})
$$

if

(1) $\operatorname{cov}(\mathscr{I}) \leq \operatorname{cov}(\mathscr{F})$ in all models of ZFC,

(2) there is at least one model of ZFC in which $\operatorname{cov}(\mathscr{I})<\operatorname{cov}(\mathscr{F})$.

This relation can be called "irreversible inequality" or "consistently strict inequality."

We remark that apart from $=, \leq$, and $\prec$ there is a fourth relation between cardinal invariants, which, for example, $\operatorname{cov}(\mathcal{N})$ and $\operatorname{cov}(\mathcal{M})$ satisfy: incomparability, namely that 
neither $\operatorname{cov}(\mathscr{I}) \leq \operatorname{cov}(\mathscr{F})$ nor $\operatorname{cov}(\mathscr{F}) \leq \operatorname{cov}(\mathscr{I})$ are provable in ZFC, or, equivalently, that there are some models of ZFC in which $\operatorname{cov}(\mathscr{I})<\operatorname{cov}(\mathscr{F})$ and other models in which $\operatorname{cov}(\mathscr{F})<\operatorname{cov}(\mathscr{I})$.

\section{Covering numbers of convexity ideals}

We return now from the short guided tour in mathematical logic to convexity ideals and see what can be said about them in the expanded context that was set in the previous section.

We now show that none of the reductions we proved in Section 2.3 are reversible. What does this mean in practice? That whatever effort, ingenuity, or technique one may invest in trying to prove, say, that $\mathscr{I}_{d} \leq \mathscr{I}_{d+1}$, the attempt will not be successful. Why? Because an acceptable proof can be transformed into a formal proof, and there is no such formal proof-if ZFC is consistent.

Theorem 4.1. For every $d \geq 1$, it holds that $\operatorname{cov}\left(\mathscr{I}_{d+1}\right) \prec \operatorname{cov}\left(\mathscr{I}_{d}\right)$.

For establishing this, one should, for every $d$, provide a model in which $\operatorname{cov}\left(\Phi_{d+1}\right)<$ $\operatorname{cov}\left(\Phi_{d}\right)$. Something better may be done.

Theorem $4.2[10]$. For every $d \geq 3$, there is a model of set theory in which $\mathfrak{c}>\operatorname{cov}\left(\Phi_{3}\right)>$ $\cdots>\operatorname{cov}\left(\mathscr{I}_{d}\right)$.

There is also a model [4] in which $\mathfrak{c}>\operatorname{cov}\left(\Phi_{2}\right)$ and a model in which $\operatorname{cov}\left(\Phi_{2}\right)>\operatorname{cov}\left(I_{3}\right)$.

We know that $\Phi_{d+1} \leq \mathscr{I}(S)$ for all closed $S \subseteq \mathbb{R}^{d}$, but it could still be the case that there are two convexity ideals of closed subsets of $\mathbb{R}^{d}$ which are incomparable or satisfy the relation $\prec$. However, we have no example of two incomparable convexity ideals. In other words, we have no evidence that the set of all convexity ideals of closed subsets in $\mathbb{R}^{d}$ for all $d \geq 1$ is not linearly ordered by $\leq$.

Theorem 4.3 (Geschke [6]). For each $d$, there is a model of ZFC in which $\operatorname{cov}\left(I_{d+1}\right)<$ $\operatorname{cov}(\Phi(S))$ for all closed uncountably convex $S \subseteq \mathbb{R}^{d}$.

Geschke's proof utilizes a geometric property common to all convexity ideals in $\mathbb{R}^{d}$ to separate them from $\mathscr{I}_{d+1}$.

The results quoted so far, show that Figure 2.2 is indeed the right figure for the relation of irreversible inequality.

It is still open whether there may be in $\mathbb{R}^{d}$ a closed set whose convexity ideal is not equivalent to $\mathscr{I}_{d^{\prime}}$ for some $d^{\prime} \leq d$. For all we know, in $\mathbb{R}^{d}$ there may just be the $d$ inequivalent convexity ideals we have listed, which are linearly ordered by irreversible reducibility.

Even if there are convexity ideals in $\mathbb{R}^{d}$ which were not spotted yet, the following weaker statement, phrased in the language of cardinal invariants and formal consistency, may still be true.

Conjecture 4.4 (the dimension conjecture). There may be $d$, but no more than $d$ different uncountable convexity numbers of closed subsets of $\mathbb{R}^{d}$ in a single model of set theory.

In dimension $d>2$, this conjecture is "open at both ends." First, it is not known if one can get all covering numbers of $\mathscr{I}_{1}$ through $\mathscr{I}_{d}$ to be different than each other in a single 
model of ZFC. This is a problem in the technology of forcing. So at the moment only $d-1$ different numbers are attained for $d>2$.

At the other end, there is no known classification of convexity ideals in $\mathbb{R}^{d}$ for $d>1$. This problem is geometric. Also, there is no upper bound on the number of different covering numbers of convexity ideals in $\mathbb{R}^{d}$ for $d>2$.

\section{Convexity ideals in $\mathbb{R}^{2}$, Ramsey ideals, and functions ideals}

The dimension conjecture has been proved for $d=2$. The proof does not determine the structure of convexity ideals in $\mathbb{R}^{2}$ under $\prec$. It utilizes the fact that convexity ideals in $\mathbb{R}^{2}$ which are not equivalent to $\Phi_{1}$ are sandwiched between Ramsey ideals from above, and ideals of continuous functions on $2^{\mathbb{N}}$, and the ideal of $\sigma$-compact subsets of $\mathbb{N}^{\mathbb{N}}$ from below.

The important property of functions ideals is that their covering numbers are either $2^{\mathrm{N}_{0}}$ or the immediate predecessor of $2^{\mathrm{N}_{0}}$; the important property about Ramsey ideals is that each of them is $\prec \mathscr{I}_{1}$. Apart from settling the dimension conjecture for $d=2$, Ramsey ideals and functions ideals have very appealing properties and are related to both analysis and graph theory. We will survey now the relations among those types of ideals. A complete picture of what is known is presented in Figure 5.1.

We begin by introducing the players.

5.1. Ramsey ideals. We start by generalizing the definition of the ideal $\Phi_{2}$. Observe that the two-place function $\Delta$ is symmetric and satisfies that if $\Delta(x, y)=d$ and $\min \left\{\Delta\left(x, x^{\prime}\right)\right.$, $\left.\Delta\left(y, y^{\prime}\right)\right\} \geq d$, then $\Delta\left(x^{\prime}, y^{\prime}\right)=d$. In particular, if parity $(\Delta(x, y))=i, i \in\{0,1\}$ and $x^{\prime}$ is sufficiently close to $x, y^{\prime}$ sufficiently close to $y$, then parity $\left(\Delta\left(x^{\prime}, y^{\prime}\right)\right)=i$ as well. This is a continuity condition, which we now state precisely.

Definition 5.1. For a topological space $X$ let $[X]^{2}$ denote the quotient of the product topology on $X^{2} \backslash\{(x, x): x \in X\}$ over the equivalence relation $(x, y) \sim(y, x)$.

A function $c:[X]^{2} \rightarrow\{0,1\}$ is called a continuous coloring if $c$ is continuous with respect to the topology on $[X]^{2}$.

Definition 5.2. Suppose that $c:[X]^{2} \rightarrow\{0,1\}$ is a continuous coloring. A subset $Y \subseteq X$ is called $c$-homogeneous if $c \uparrow[Y]^{2}$ is constant. Let $\Phi_{c}$ be the $\sigma$-ideal $\sigma$-generated by all $c$-homogeneous subsets of $X$.

We define $c_{\min }:\left[2^{\mathbb{N}}\right]^{2} \rightarrow\{0,1\}$ by $c_{\min }(x, y)=\operatorname{parity}(\Delta(x, y))$. This is a continuous coloring. Furthermore, the definition of $\Phi_{2}$ gives us immediately that

$$
\mathscr{I}_{c_{\min }}=\Phi_{2}
$$

Fact 5.3. Suppose $X$ is a Polish space and $c:[X]^{2} \rightarrow\{0,1\}$ is a continuous coloring. Then $\Phi_{c}$ is proper if and only if there is a perfect $P \subseteq X$ so that $M(P) \leq \mathscr{I}_{c}$.

Proof. One direction is trivial. For the other direction, suppose that $\Phi_{c}$ is proper. Let $A$ be the union of all open sets which belong to $\Phi_{c}$. Then $A$ is open and belongs itself to $\mathscr{I}_{c}$. Let $P=X \backslash A$. Clearly, $P$ nonempty and perfect. The continuity of $c$ implies that 
(6)

$$
\left(\operatorname{cov}\left(\operatorname{Cont}\left(2^{\omega}\right)\right)\right)^{+}
$$

(5)

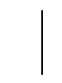

5)

(4)

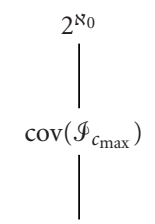

(3)

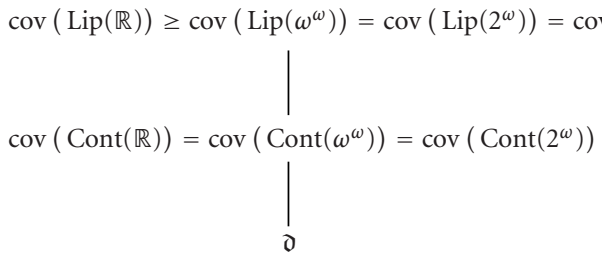

Figure 5.1

a closure of a $c$-homogeneous set is $c$-homogeneous, thus every $c$-homogeneous subset of $P$ is nowhere dense. Now the identity function on $P$ is a reduction of $M(P)$ to $\mathscr{I}_{c}$.

From now on we will assume, by replacing a given $c:[X]^{2} \rightarrow\{0,1\}$ by $c: \uparrow[P]^{2}$, that all continuous colorings we consider satisfy that no open set is homogeneous.

Fact 5.4. Suppose $X$ is a perfect Polish space and $c:[X]^{2} \rightarrow\{0,1\}$ has no open homogeneous set. Then $\mathscr{I}_{c_{\min }} \leq \mathscr{I}_{c}$.

The proof is straightforward.

Theorem 5.5 [5]. There exists a continuous pair-coloring $c_{\max }: 2^{\mathbb{N}} \rightarrow\{0,1\}$ so that for all continuous pair-coloring $c: X \rightarrow\{0,1\}$ with no open homogeneous sets on some Polish space $X$, it holds that

$$
\operatorname{cov}\left(\mathscr{T}_{c_{\min }}\right) \leq \operatorname{cov}\left(\mathscr{I}_{c}\right) \leq \operatorname{cov}\left(\mathscr{I}_{c_{\max }}\right) .
$$

It is interesting to point out that $c_{\max }$ is defined on a compact space.

TheOREM 5.6. There is a model of ZFC in which $\operatorname{cov}\left(\mathscr{I}_{c_{\max }}\right)<2^{\mathrm{N}_{0}}$. In particular, $\mathscr{I}_{c_{\max }} \prec \mathscr{I}_{1}$ and consequently $\Phi_{c} \prec \Phi_{1}$ for every continuous pair-coloring c on a Polish space.

5.2. Functions ideals over the square. Let $X$ be any infinite set. Let $\operatorname{Func}(X)$ be the ideal generated over $X^{2}$ by all graphs and inverses of graphs of functions from $X$ to $X$.

A covering collection in $\operatorname{Func}(X)$ is a set $\mathscr{F}$ of functions from $X$ to $X$ so that for all $\left(x_{1}, x_{2}\right) \in X^{2}$, there is $f \in \mathscr{F}$ so that $f\left(x_{1}\right)=x_{2}$ or $f\left(x_{2}\right)=x_{1}$.

Theorem 5.7 (Sierpiński). For every infinite cardinal $\aleph_{\alpha+1}$, it holds that $\operatorname{cov}\left(\operatorname{Func}\left(\aleph_{\alpha+1}\right)\right)=$ $\mathrm{\aleph}_{\alpha}$ and if $\alpha$ is limit, then $\operatorname{cov}\left(\operatorname{Func}\left(\mathrm{\aleph}_{\alpha}\right)\right)=\mathrm{\aleph}_{\alpha}$. 
In particular, there are countably many functions $f_{n}: \aleph_{1} \rightarrow \aleph_{1}$ so that for all $\alpha, \beta \in \aleph_{1}$, there is some $n$ so that $f_{n}(\alpha)=\beta$ or $f_{n}(\beta)=\alpha$. Thus, the following holds.

Corollary 5.8. The CH implies that $\operatorname{cov}(\operatorname{Func}(\mathbb{R}))=\aleph_{0}$.

In the case $\mathrm{CH}$ holds, $\operatorname{Func}(\mathbb{R})$ is not contained in a proper $\sigma$-ideal over $\mathbb{R}$.

The graph of a continuous function from $\mathbb{R}$ to $\mathbb{R}$ is, though, a nowhere dense subset of $\mathbb{R}^{2}$. Thus the graphs and inverses of graphs of continuous real functions do generate a proper $\sigma$-ideal over $\mathbb{R}^{2}$.

Definition 5.9. Suppose $X$ is a metric space. Then $\operatorname{Cont}(X)$ is the $\sigma$-ideal which is $\sigma$ generated over $X^{2}$ by graphs and inverses of graphs of continuous functions from $X$ to $X$. Lip $(X)$ is the $\sigma$-ideal which is $\sigma$-generated over $X^{2}$ by graphs and inverses of graphs of Lipschitz continuous functions.

Since $\operatorname{Lip}(X) \subseteq \operatorname{Cont}(X) \subseteq \operatorname{Func}(X)$ for every metric space $X$, one has trivially

$$
\operatorname{cov}(\operatorname{Lip}(X)) \geq \operatorname{cov}(\operatorname{Cont}(X)) \geq \operatorname{cov}(\operatorname{Func}(X)) .
$$

Hence, by Sierpiński's theorem we have the following important fact.

Fact 5.10. For every perfect Polish space $X$,

$$
(\operatorname{cov}(\operatorname{Cont}(X)))^{+} \geq \max \left\{\aleph_{2}, 2^{\aleph_{0}}\right\}
$$

Proof. Since $\operatorname{cov}(\operatorname{Cont}(X)) \geq \mathfrak{N}_{1}$, it holds that $(\operatorname{Cov}(\operatorname{Cont}(X)))^{+} \geq \mathfrak{\aleph}_{2}$ for every perfect Polish $X$. That $(\operatorname{cov}(\operatorname{Cont}(X)))^{+} \geq 2^{\mathrm{\aleph}_{0}}$ follows directly from Sierpiński's theorem and $|X|=2^{\mathrm{N}_{0}}$.

The relation between functions ideals and convexity ideals is set via the following.

Theorem 5.11. $\operatorname{Lip}\left(2^{\mathbb{N}}\right)$ and $\mathscr{I}_{2}$ are equivalent.

By what we have seen so far, $\mathscr{I}_{2}$ plays three different roles: it is a convexity ideal in $\mathbb{R}^{2}$, it is the bottom (with respect to reducibility) Ramsey ideal, and is also (equivalent to) the Lipschitz ideal $\operatorname{Lip}\left(2^{\mathbb{N}}\right)$. We remark that $\mathscr{I}_{2}$ is actually isomorphic to the ideal $\operatorname{Lip}_{1,1 / 2}\left(2^{\mathbb{N}}\right)$ which is $\sigma$-generated over $\left(2^{\mathbb{N}}\right)^{2}$ by all graphs of functions satisfying the Lipschitz condition with Lipschitz constant 1 and all inverses of graphs of functions which satisfy the Lipschitz condition with Lipschitz constant 1/2 (see [5]).

We quote the following result from [5].

Theorem 5.12. There is a model of ZFC in which $\operatorname{cov}(\operatorname{Cont}(\mathbb{R}))=\kappa_{1}$ and $\operatorname{cov}(\operatorname{Lip}(\mathbb{R}))=$ $\aleph_{2}$. Therefore $\operatorname{Cont}(\mathbb{R}) \prec \operatorname{Lip}(\mathbb{R})$.

We remark that by Steprāns' [15] also the $\sigma$-ideal $\sigma$-generated over $\mathbb{R}^{2}$ by $C_{1}$ functions is not equivalent to $\mathscr{I}_{1}$. However, the $\sigma$-ideal which is $\sigma$-generated over $\mathbb{R}^{2}$ by twice differentiable function is equivalent to $\Phi_{1}$ by the following result from [1].

Theorem 5.13 [1]. There exists a differentiable function $f: \mathbb{R} \rightarrow \mathbb{R}$ and an infinite perfect set $P \subseteq \mathbb{R}$ such that the derivative of $f$ is constantly 0 on $P$ and no function which is twice differentiable intersects $f \uparrow P$ in infinitely many points. 
Since the derivative of $f$ is 0 on $P$, no inverse of a differentiable function intersects $f \uparrow P$ in more than one point. Thus, $f \uparrow P$ itself is a reduction of the $\sigma$-ideal $\sigma$-generated over $\mathbb{R}^{2}$ by graphs of twice differentiable and inverses of graphs of differentiable functions to the ideal of countable subsets of $P$.

5.3. $\sigma$-compact subsets of the irrationals. Finally, we denote the $\sigma$-ideal which is $\sigma$ generated over $\mathbb{N}^{\mathbb{N}}$ by all compact subsets of $\mathbb{N}^{\mathbb{N}}$ by $\operatorname{Comp}\left(\mathbb{N}^{\mathbb{N}}\right)$. The covering number of this ideal is denoted by $\mathfrak{d}$.

The following crucial inequality does not follow from a reduction of $\operatorname{Cont}\left(2^{\mathbb{N}}\right)$ to $\operatorname{Comp}\left(\mathbb{N}^{\mathbb{N}}\right)$. In fact, it is known that no such definable reduction exists.

Theorem 5.14. $\mathfrak{d} \leq \operatorname{cov}\left(\operatorname{Cont}\left(2^{\omega}\right)\right)$.

Proof. Rather than showing that there is some $f$ that takes every cover of $2^{\mathbb{N}} \times 2^{\mathbb{N}}$ by continuous functions to a compact cover of $\mathbb{N}^{\mathbb{N}}$, we will show something weaker: that for every cover of $2^{\mathbb{N}}$ by continuous functions, there is map $F$ that takes the elements of the cover to a compact cover of $\mathbb{N}^{\mathbb{N}}$.

We utilize the fact that the subspace of $2^{\mathbb{N}}$ consisting of all sequences with infinitely many 1's is homeomorphic to $\mathbb{N}^{\mathbb{N}}$. Via an identification of this subspace with $\mathbb{N}^{\mathbb{N}}$, we have $\mathbb{N}^{\mathbb{N}} \subseteq 2^{N}$ and $2^{\mathbb{N}} \backslash \mathbb{N}^{\mathbb{N}}$ is countable, since there are only countably many eventually zero sequences in $2^{\mathbb{N}}$.

Suppose that $\left\{f_{\alpha}: \alpha \in I\right\}$ is a set of continuous self-maps on $2^{\mathbb{N}}$ such that for all $x, y \in$ $2^{\mathbb{N}}$ there is some $\alpha \in I$ so that either $f_{\alpha}(x)=y$ or $f_{\alpha}(y)=x$.

Case 1. $\left\{f_{\alpha}(q): q \in 2^{\mathbb{N}} \backslash \mathbb{N}^{\mathbb{N}}, \alpha \in I\right\} \supseteq \mathbb{N}^{\mathbb{N}}$. In this case, let $F(f)=\left\{f(q): q \in 2^{\mathbb{N}} \backslash \mathbb{N}^{\mathbb{N}}\right\} \cap$ $\mathbb{N}^{\mathbb{N}}$ for every continuous $f: 2^{\mathbb{N}} \rightarrow 2^{\mathbb{N}}$. Clearly, $\bigcup\left\{F\left(f_{\alpha}\right): \alpha \in I\right\}=\mathbb{N}^{\mathbb{N}}$.

Case 2. There is some $x \in \mathbb{N}^{\mathbb{N}}$ so that $x \neq f_{\alpha}(q)$ for all $\alpha \in I$ and $q \in 2^{\mathbb{N}} \backslash \mathbb{N}^{\mathbb{N}}$. In this case let $F(f)=f^{-1}(x) \cup\left(\{f(x)\} \cap \mathbb{N}^{\mathbb{N}}\right)$ if $f^{-1}(x) \subseteq \mathbb{N}^{\mathbb{N}}$ and let $F(f)=\{x\}$ otherwise. For every continuous self map $f$ of $2^{\mathbb{N}}, f^{-1}(x)$ is a compact subset of $2^{\mathbb{N}}$; in the case $f^{-1}(x) \subseteq \mathbb{N}^{N}, f^{-1}(x)$ is a compact subset of $\mathbb{N}^{\mathbb{N}}$ and so is $F(f)=f^{-1}(x) \cup\left(\{f(x)\} \cap \mathbb{N}^{\mathbb{N}}\right)$. In the other case $F(f)$ is a singleton, and hence compact.

To see that $\bigcup\left\{F\left(f_{\alpha}\right): \alpha \in I\right\}=\mathbb{N}^{\mathbb{N}}$ just recall that for every $y \in 2^{\mathbb{N}}$, there is some $\alpha$ for which $f_{\alpha}(x)=y$ or $f_{\alpha}(y)=x$.

Figure 5.1 summarizes the relations between the ideals we introduced.

The relation between every cardinal invariant in the figure to every cardinal invariant in any line above it is irreversible inequality-with one exception, that of $\operatorname{cov}(\operatorname{Lip}(\mathbb{R}))$. Not all of the inequalities in this figure were proved by providing a reduction. A good part of the inequalities were proved indirectly by more abstract means. For details see [5].

An inequality that was recently proved by Geschke, and which is not present in the figure, is $\operatorname{cov}(\operatorname{Lip}(\mathbb{R})) \leq \operatorname{cov}\left(\mathscr{I}_{c_{\max }}\right)$.

For each of the lines (1)-(4) in the figure there is a model of ZFC in which all cardinals in that line are equal to $\kappa_{1}$ and on the line above are equal to $\kappa_{2}$, provided $\operatorname{cov}(\operatorname{Lip}(\mathbb{R})$ ) is removed from line (3). The difference between line (2) and line (6) is at most one cardinal.

It is the inequality between $\mathfrak{d}$ and $\operatorname{cov}\left(\Phi_{c_{\text {min }}}\right)$ that enables the proof of Theorem 5.5.

Proofs can be found in [5]. 
5.4. The dimension conjecture in $\mathbb{R}^{2}$. The following geometric theorem connects convexity ideals in $\mathbb{R}^{2}$ to Ramsey ideals.

THEOREm 5.15 [4]. For every closed, uncountably convex $S \subseteq \mathbb{R}^{2}$, either there is a perfect $P \subseteq S$ so that $|c \cap P|<3$ for all convex $c \subseteq S$ (and then $\Phi_{1} \leq \mathscr{I}(S)$ ), or else then there is a partition $\operatorname{dom} \Phi(S)=\bigcup A_{n}$ so that $A_{n}$ is homeomorphic to $\mathbb{N}^{\mathbb{N}}$ for each $n$ and $\mathscr{I}(S)\left\lceil A_{n}=\right.$ $\Phi_{c_{n}}$ for some continuous coloring $c_{n}:\left[A_{n}\right]^{2} \rightarrow\{0,1\}$.

By the last theorem, for every closed and uncountably convex $S \subseteq \mathbb{R}^{2}$,

$$
\operatorname{cov}\left(\mathscr{I}_{2}\right)=\operatorname{cov}\left(\mathscr{I}_{c_{\min }}\right) \leq \operatorname{cov}(\mathscr{I}(S)) \leq \operatorname{cov}\left(\mathscr{I}_{c_{\max }}\right) .
$$

Namely, the convexity number of each closed uncountably convex subset of $\mathbb{R}^{2}$ is sandwiched between lines (3) and (4) in the Figure 5.1. Since the cardinals in those lines are either equal or that in line (4) is the successor of that in line (3), we see that there cannot be more than two uncountable convexity numbers of closed subsets of $\mathbb{R}^{2}$.

On the other hand, since $\mathscr{I}_{2}$ and $\mathscr{I}_{1}$ are realized as the convexity ideal of some closed subsets in the plane, and $\mathscr{I}_{2} \prec \Phi_{1}$, there are models in which two different uncountable convexity numbers occur in $\mathbb{R}^{2}$.

Thus, the following holds.

THEOREM 5.16. There are models of ZFC with two, but no models of ZFC with more than two, uncountable convexity numbers of closed subsets of $\mathbb{R}^{2}$.

Let $S \subseteq \mathbb{R}^{2}$ be a closed set. A set $P \subseteq S$ is called a 3 -clique if for all distinct $x_{1}, x_{2}, x_{3} \in$ $P$ it holds that $\operatorname{conv}\left(x_{1}, x_{2}, x_{3}\right) \nsubseteq S$. If $S \subseteq \mathbb{R}^{2}$ contains a perfect 3 -clique, then clearly $\operatorname{cov}(\Phi(S))=2^{\mathrm{N}_{0}}$ in all models of ZFC. The following is a characterization of nonexistence of perfect 3-cliques by metamathematical means.

Theorem 5.17. Suppose $S \subseteq \mathbb{R}^{2}$ is closed. Then there is no perfect 3-clique $P \subseteq S$ if and only if $\operatorname{cov}(\mathscr{I}(S)) \prec 2^{\mathrm{N}_{0}}$.

\section{Concluding remarks}

We remark first that although cardinal invariants of the continuum are studied extensively in set theory, most of the invariants that set theorists have studied have no connection with Euclidean dimension. The study of convexity ideals and their relation to Euclidean dimension is an atypical case in which both set-theoretic techniques and Euclidean dimension play each a substantial role.

Second, since each of the $\sigma$-ideals that have been treated here is a subideal of the meager ideal, one may wonder whether reducibility between $\sigma$-ideals should not actually be studied in the larger generality of all $\sigma$-subideals of the meager ideal. The fact is, though, that classification of $\sigma$-subideals of the meager ideal is impossible. Even the classification of all $\sigma$-subideals of the meager ideal that have particularly "nice" definitions is impossible, for the following reason. Answering a question of Blass, who asked if all covering numbers of $\sigma$-ideal which have a particularly simple definition could be classified [3], Goldstern and Shelah [7] produced an uncountable list $\left\langle\Phi_{\alpha}: \alpha<\omega_{1}\right\rangle$ of very simply defined $\sigma$-subideals of the meager ideal, called slalom ideals, with the following remarkable 
property: for every list $\left\langle\lambda_{\alpha}: \alpha<\omega_{1}\right\rangle$ of regular uncountable cardinals, there is a model of ZFC in which $\operatorname{cov}\left(\mathscr{I}_{\alpha}\right)=\lambda_{\alpha}$. This is a very strong negative result about classification. Not only one cannot prove the existence of any reduction of $\mathscr{I}_{\alpha}$ to $\mathscr{I}_{\beta}$ if $\alpha \neq \beta$, but even conditional information is not possible: even if one knows all covering numbers of all $\Phi_{\alpha}$ except for $\alpha=\alpha_{0}$, still nothing can be deduced about $\operatorname{cov}\left(\mathscr{I}_{\alpha_{0}}\right)$.

In contrast to the chaos which rules the realm of all subideals of the meager ideal-as the Goldstern-Shelah result exemplifies-the geometry of $\mathbb{R}^{d}$ carves out of the nonclassifiable wealth of simple $\sigma$-subideals of the meager ideal a corner in which classification is possible.

Finally, convexity ideals in $\mathbb{R}^{2}$ display another peculiar behavior: they all sit above the Lipschitz functions ideal $\operatorname{Lip}\left(2^{\mathbb{N}}\right)$. This leaves a very narrow window for their covering numbers: they can be either the continuum or its immediate successor. Still, they are not reducible to $\mathscr{I}_{1}$. Thus, a natural problem arises.

Question 6.1. Among all nicely defined $\sigma$-subideals of the meager ideal which are not reducible to $\Phi_{1}$, is there one with a largest covering number?

At the moment, $\mathscr{I}_{c_{\max }}$ is a candidate. Another candidate is the $\sigma$-ideal which is $\sigma$ generated over $\mathbb{R}^{2}$ by all graphs and inverses of graphs of $C_{1}$ real functions.

6.1. The final word on methodology. Formal independence over ZFC is a syntactic relation studied by logicians; why should a mathematician who works in analysis or in convex geometry bother with it? There are several answers.

First, formal independence provides a tool for testing that a certain theorem is the best possible. For instance, Theorem 5.13 (which was proved by analysts) cannot be improved from twice differentiable functions to continuously differentiable functions because of Steprāns' consistency result [15].

Second, when reaching the limit of knowledge of what is true, using formal consistency one can proceed to find out the broader picture of what is possibly true. The relation of irreversible reducibility under which convexity ideal in $\mathbb{R}^{d}$ forms a nice pattern is such an example. Formal independence over ZFC has created a situation in which one must look at a variety of different models of ZFC rather than at a single one to fully understand a phenomenon.

This way of looking at things is orthogonal to the philosophical quibble of what should be true. To look at models in which the $\mathrm{CH}$ holds, or in which the $\mathrm{CH}$ fails, for the purpose of finding out that a certain reduction may not exist, one does not need to have an opinion on whether the $\mathrm{CH}$ should be accepted as true. (The information one gathers this way, though, may help a mathematician in adopting a position as to what "should" or "should not" be accepted as true. It is interesting to point out that Kurt Gödel, in his 1947 paper What is Cantor's continuum problem [7], rejected the CH, 17 years before Cohen's proof of the formal independence of $\mathrm{CH}$, on the basis that it did not have "verifiable consequences," namely, that it had many consequences, none of which lead to easy proof of "really true" facts. Later on it turned out that all the examples of consequences of $\mathrm{CH}$ that Gödel quoted, were indeed independent of ZFC.)

Finally, formal independence and cardinal invariants provide a rich language for discussing small sets in $\mathbb{R}^{d}$, and enable the discovery of geometric properties of $\mathbb{R}^{d}$ which are 
not accessible otherwise. The dimension conjecture and its proof for $d=2$ is one such example, and the characterization of 3-cliques in a closed planar set (see Theorem 5.17) is another.

\section{Acknowledgments}

This work is partially supported by an ISF Grant. The author wishes to thank the referees for several helpful comments and suggestions.

\section{References}

[1] S. Agronsky, A. M. Bruckner, M. Laczkovich, and D. Preiss, Convexity conditions and intersections with smooth functions, Trans. Amer. Math. Soc. 289 (1985), no. 2, 659-677.

[2] T. Bartoszyński, Measure and Category Invariants, to appear in Handbook of Set Theory.

[3] A. Blass, Simple cardinal characteristics of the continuum, Set Theory of the Reals (Ramat Gan, 1991), Israel Math. Conf. Proc., vol. 6, Bar-Ilan University, Ramat Gan, 1993, pp. 63-90.

[4] K. Gödel, Die Vollständigkeit des Axiome dere logischen Funktionenkaküls, Monatshefte für Mathematik und Physik 37 (1930), 349-360.

[5] _ Über formal unentschindbare Sätze der Principia Mathematica und verwandter Systeme I, Monatshefte für Mathematik und Physik 38 (1931), 173-198.

[6] - The Consistency of the Continuum Hypothesis, Annals of Mathematics Studies, no. 3, Princeton University Press, New Jersey, 1940.

[7] W What is Cantor's continuum problem,? Amer. Math. Monthly 54 (1947), no. 9, 515525.

[8] S. Geschke, More on convexity numbers of closed sets in $\mathbb{R}^{n}$, Proc. Amer. Math. Soc. 133 (2005), no. $5,1307-1315$.

[9] S. Geschke, M. Goldstern, and M. Kojman, Continuous Ramsey theory on Polish spaces and covering the plane by functions, J. Math. Log. 4 (2004), no. 2, 109-145.

[10] S. Geschke and M. Kojman, Convexity numbers of closed sets in $R^{n}$, Proc. Amer. Math. Soc. 130 (2002), no. 10, 2871-2881.

[11] S. Geschke, M. Kojman, W. Kubiś, and R. Schipperus, Convex decompositions in the plane and continuous pair colorings of the irrationals, Israel J. Math. 131 (2002), 285-317.

[12] M. Goldstern and S. Shelah, Many simple cardinal invariants, Arch. Math. Logic 32 (1993), no. 3, 203-221.

[13] A. S. Kechris, Classical Descriptive Set Theory, Graduate Texts in Mathematics, vol. 156, Springer, New York, 1995.

[14] M. Kojman, M. A. Perles, and S. Shelah, Sets in a Euclidean space which are not a countable union of convex subsets, Israel J. Math. 70 (1990), no. 3, 313-342.

[15] J. Steprāns, Decomposing Euclidean space with a small number of smooth sets, Trans. Amer. Math. Soc. 351 (1999), no. 4, 1461-1480.

Menachem Kojman: Department of Mathematics, Ben Gurion University of the Negev, Beer Sheva 84105, Israel

E-mail address: kojman@math.bgu.ac.il 


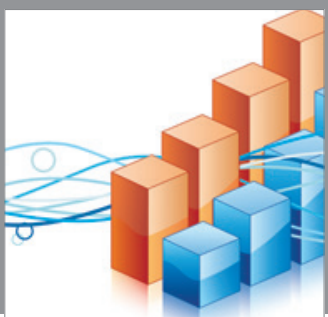

Advances in

Operations Research

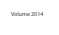

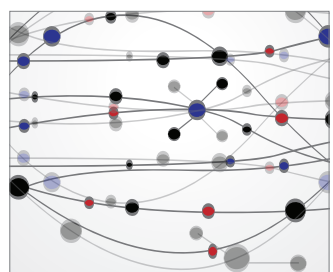

\section{The Scientific} World Journal
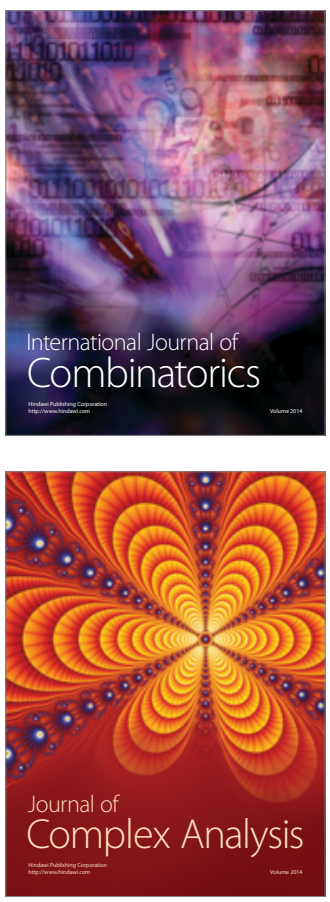

International Journal of

Mathematics and

Mathematical

Sciences
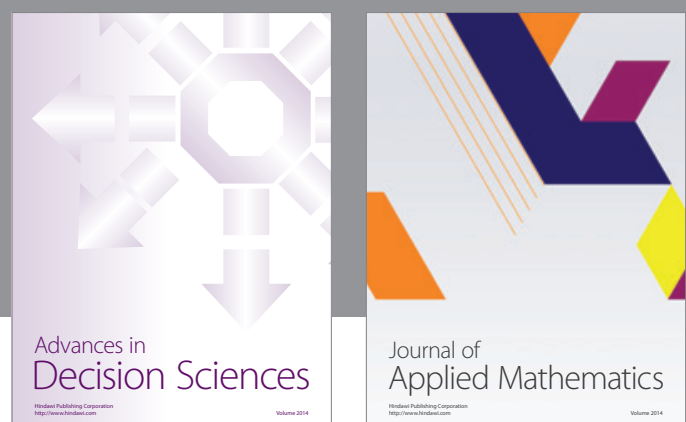

Journal of

Applied Mathematics
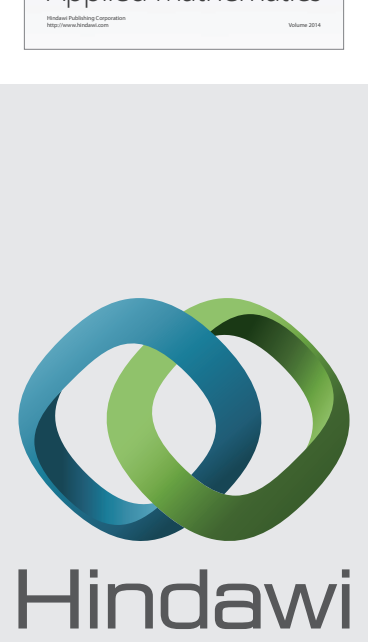

Submit your manuscripts at http://www.hindawi.com
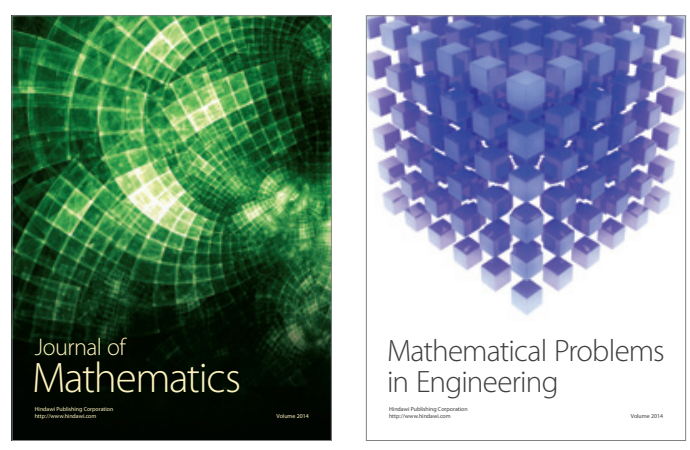

Mathematical Problems in Engineering
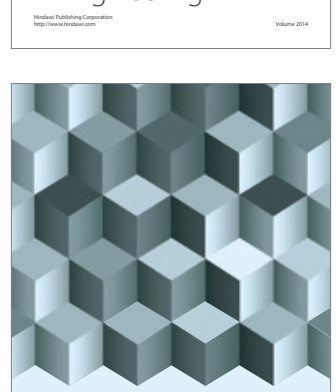

Journal of

Function Spaces
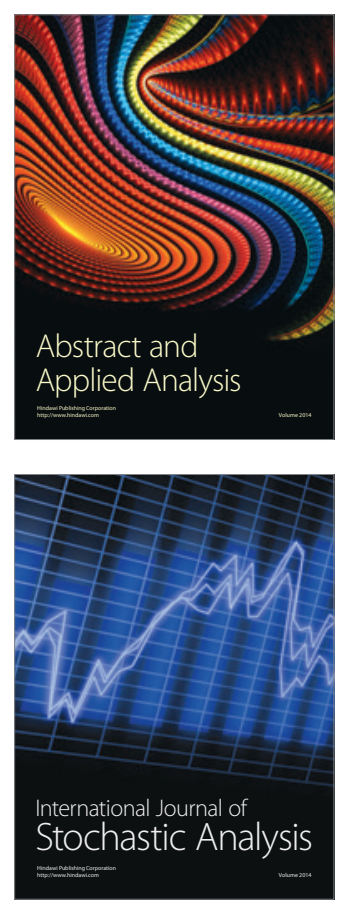

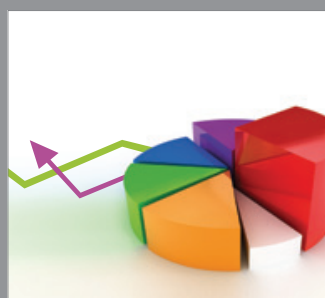

ournal of

Probability and Statistics

Promensencen
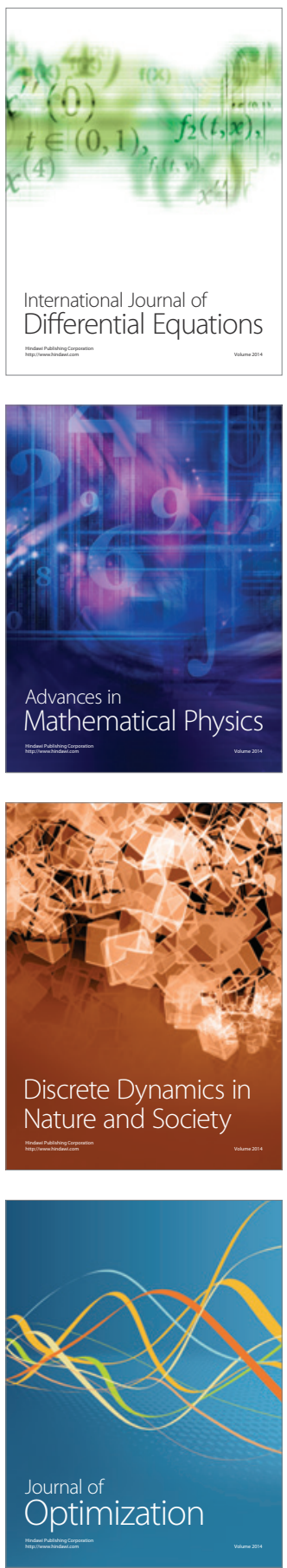